Les Cahiers du Gres

\section{Cohabitation interculturelle, pratique religieuse et espace urbain : quelques réflexions à partir du cas des communautés hassidiques juives d'Outremont/Mile-End1}

Julie Elizabeth Gagnon
Les Cahiers du Gres Série Diversité urbaine Groupe de Recherche Ethricte et Societ

Volume 3, numéro 1, printemps 2002

URI : https://id.erudit.org/iderudit/009429ar

DOI : https://doi.org/10.7202/009429ar

Aller au sommaire du numéro

Éditeur(s)

Groupe de recherche ethnicité et société

CEETUM

ISSN

1499-0431 (imprimé)

1499-044X (numérique)

Découvrir la revue

Citer cet article

Gagnon, J. E. (2002). Cohabitation interculturelle, pratique religieuse et espace urbain : quelques réflexions à partir du cas des communautés hassidiques juives d'Outremont/Mile-End1. Les Cahiers du Gres, 3(1), 39-53.

https://doi.org/10.7202/009429ar d'utilisation que vous pouvez consulter en ligne. 


\section{Julie Elizabeth GAGNON}

Candidate au doctorat

Département d'études urbaines

INRS Urbanisation, Culture et Société, Montréal

Cohabitation interculturelle, pratique religieuse et espace urbain : quelques réflexions à partir du cas des communautés hassidiques juives d'Outremont/Mile-End ${ }^{1}$

Cet article vise à présenter quelques éléments de réflexion sur l'aménagement de lieux de culte minoritaires ${ }^{2}$ dans la région de Montréal, dans une perspective de cohabitation interculturelle. La pertinence d'étudier les lieux de culte minoritaires relève tout d'abord de l'intérêt de la dimension religieuse pour aborder la question des rapports à la différence et, par le biais des pratiques culturelles et religieuses, de son impact sur la vie quotidienne et la cohabitation interculturelle. Par ailleurs, depuis les années 1980, la radicalisation politique de certains mouvements à caractère religieux sur la scène internationale suscite des remises en question quant à l'importance des identités culturelles, et notamment religieuses, dans les problématiques géopolitiques actuelles. Les évènements terroristes perpétrés aux États-Unis le 11 septembre dernier, attribués à des groupes radicaux islamistes, illustrent de façon tragique la pertinence de la variable religieuse pour comprendre certains développements sociaux contemporains.

Dans les années 1990, à l'instar de la situation dans les provinces anglophones du Canada, plusieurs incidents sont venus remettre en question la neutralité présumée de la société québécoise en ce qui concerne les différences religieuses. On pense notamment à l'af- faire du hijab (Ciceri, 1998), au débat sur la religion à l'école (McAndrew, 1996 ; Milot \& Proulx, 1999, etc.) et, plus récemment, à la question de l'erouv à Outremont et des controverses au sujet de l'agrandissement d'une synagogue dans le Mile-End. Nous verrons ainsi comment les questions liées à la religion et à la pratique religieuse refont surface lorsqu'il est question de l'immigration internationale et de l'accommodement des différences culturelles à l'échelle urbaine.

Nous commencerons par brosser un rapide tableau de l'immigration et de la diversité culturelle et religieuse dans la région de Montréal ; nous présenterons ensuite deux études de cas d'aménagement (ou de réaménagement) de lieux de culte qui nous permettront de faire quelques constats par rapport aux défis que pose cette diversité pour la cohabitation interculturelle à l'échelle locale, ainsi que du rôle du rapport à l'espace urbain dans cette dynamique.

L'immigration et la diversité culturelle dans la région montréalaise

La diversité ethnoculturelle croissante des sociétés contemporaines se traduit en contexte urbain par la cohabitation, à l'échelle locale, d'une diversité de groupes et d'individus aux origines et pratiques culturelles, sociales et religieuses parfois fort différentes. L'immigration internationale est l'un des facteurs clé contribuant à cette diversité. À Montréal, comme dans d'autres grandes villes nord-américaines, cet afflux de migration s'est non seulement intensifié au cours des deux dernières décennies, mais s'est aussi diversifié du point de vue des régions d'origine. Au recensement de 1996, les immigrants représentaient $18 \%$ de la population totale de la région métropolitaine de Montréal, et près de $25 \%$ des résidants 
de la ville de Montréal elle-même. Une diversité croissante des origines nationales et des pratiques culturelles, linguistiques et religieuses caractérise aussi la population montréalaise dans son ensemble. Bien que cette diversité culturelle ait traditionnellement été concentrée dans la ville de Montréal, on constate depuis 10 ou 15 ans un mouvement de « déconcentration » de cette population (à l'instar du mouvement de la population non-immigrante) vers les autres municipalités de l'île et vers les proches banlieues de Laval et de la Rive-Sud. Ceci fait en sorte qu'un nombre croissant de quartiers et de municipalités accueillent des immigrants sur leur territoire, et que plusieurs secteurs de la région métropolitaine deviennent de plus en plus mixtes du point de vue des origines nationales et culturelles.

De façon générale, la diversité culturelle et la présence d'immigrants s'expriment de différentes façons dans l'environnement urbain. II peut s'agir de la présence en soi de personnes dont l'apparence (vêtements, attributs physiques, etc.) ou la langue d'usage reflètent leurs origines culturelles, de commerces et de restaurants « ethniques », ou encore d'un marquage au niveau du cadre bâti par le biais d'éléments architecturaux ou d'aménagements extérieurs particuliers (les jardins méditerranéens ou les icônes religieuses des Portugais dans le quartier Mile-End, par exemple). Mais un des signes les plus visibles de la diversité est sans doute la présence de lieux de culte affiliés à des groupes immigrants ou à certaines communautés culturelles. De façon générale, la ville de Montréal compte un nombre important de lieux de culte, soit autour de 500 églises, synagogues, mosquées et temples divers ; pour sa part, le reste de l'île de Montréal en compterait environ 300 , pour un total de près de 800 lieux de culte sur le territoire qui deviendra en janvier 2002 la nouvelle Ville de Montréal $^{3}$. Même si la majorité des lieux de culte sont affiliés aux églises catholiques et protestantes établies de plus longue date, le nombre de lieux de culte se rattachant à des traditions religieuses en émergence au Québec augmente rapidement, suivant l'augmentation du nombre de leurs fidèles dans la région montréalaise. On constate à cet effet que les religions nonchrétiennes orientales (dont le bouddhisme, I'hindouisme, l'islam et le sikhisme) sont les seules à voir leurs effectifs augmenter dans la région montréalaise entre les recensements de 1981 et 1991 ; les fidèles des autres grandes traditions religieuses, soit les catholiques, les protestants ${ }^{4}$, les chrétiens orthodoxes et les Juifs voient plutôt leur nombre diminuer dans l'ensemble.

Ces différences peuvent avoir une influence sur plusieurs aspects de la vie quotidienne, des habitudes alimentaires aux préférences sur le plan du logement, en passant par les attitudes par rapport à la pudeur et la sociabilité publique. Elles impliquent aussi, à l'échelle locale, que soient élaborées des manières d'accommoder les différences afin de permettre la coexistence à l'intérieur d'un même espace.

\section{L'accommodement des différences et la cohabitation interculturelle}

Vivre dans une grande ville implique le plus souvent de côtoyer des individus ou des groupes dont on considère les origines et les pratiques culturelles (au sens anthropologique large) comme différentes des nôtres. Depuis les travaux de l'École de Chicago dans les années 1930, I'hétérogénéité (culturelle et sociale) est considérée comme une caractéristique fondamentale des sociétés urbaines. Yves Grafmeyer (1999) propose que les deux dimensions essentielles de cette hétérogénéité soient la 
distance sociale (qui renvoie aux places occupées dans la division du travail et aux hiérarchies socio-économiques qui en découlent) et l'altérité (différences liées à la nationalité, l'origine géographique ou « ethnique », aux références culturelles, à la religion, etc.). L'intersection de ces deux grands vecteurs d'hétérogénéité urbaine, combinée avec un certain nombre d'autres facteurs relevant des structures matérielles et des configurations sociodémographiques propres à chaque milieu, engendre une grande diversité d'enjeux et de modalités de coexistence à l'échelle locale. Suivant cette logique, nous partirons de l'hypothèse que les caractéristiques urbaines, sociales et démographiques des milieux locaux ont un rôle important dans la construction d'un modus vivendi qui permet l'accommodement des différences, comme le suggèrent les travaux sur les quartiers multiethniques montréalais de $\mathrm{A}$. Germain et al. (1995).

À travers l'étude de deux cas spécifiques, nous proposons dans cet article de réfléchir à la dimension spécifiquement urbaine de la cohabitation interculturelle en examinant le rôle du rapport à l'espace. Nous entendons par là la façon dont des individus et des groupes perçoivent, se représentent et investissent l'espace urbain, matériellement et symboliquement. L'étude de controverses d'aménagement impliquant des lieux de culte nous permettra ainsi d'analyser comment des groupes différents sur le plan des origines et des pratiques sociales, culturelles et religieuses, en viennent à partager (ou pas) un espace local commun. Nous présentons d'abord les principaux éléments des deux cas de controverses d'aménagement que nous avons étudiés, puis nous discuterons des principaux enjeux qui en découlent en mettant l'accent sur leur dimension spécifiquement urbaine.
COHABITATION INTERETHNIQUE, ESPACE URBAIN ET PRATIQUE RELIGIEUSE : DEUX COMMUNAUTÉS HASSIDIQUES DU MILE-ENDI OUTREMONT

La communauté juive ultraorthodoxe et hassidique montréalaise ${ }^{5}$, qui regroupe environ 6000 personnes, se concentre dans certains secteurs de la municipalité d'Outremont et des quartiers montréalais adjacents, notamment le quartier Mile-End (Shahar et al., 1997). Les régions plus centrales de ce secteur formaient jusqu'aux années 1940 ou 1950 le cœur du quartier résidentiel des immigrants juifs montréalais. Parmi les premiers Juifs hassidiques à venir s'installer à Montréal étaient des survivants de l'Holocauste en provenance d'Europe de l'Est, arrivés dans les années suivant la fin de la Seconde Guerre mondiale. En effet, avant la fin des années 1950, le secteur ne comptait que peu de Juifs hassidiques ou même ouvertement religieux, bon nombre des Juifs montréalais de l'époque étant séculiers, voire d'allégeance politique gauchiste et ne s'identifiant pas avec les Juifs « religieux ». Le caractère « orthodoxe » de la communauté juive locale commence seulement à s'affirmer dans les années 1980, prenant racine dans la croissance démographique rapide de cette population et amplifié par le départ des familles juives plus " séculières » ou moins pratiquantes vers les banlieues plus à l'ouest au cours des années 1940-506. Aujourd'hui, la grande majorité des Juifs hassidiques et ultraorthodoxes vivent dans le secteur MileEnd/bas-Outremont et quelques secteurs adjacents de la ville de Montréal?.

La communauté juive hassidique de Montréal est en fait composée de plusieurs sous-groupes se définissant à partir de leur degré d'orthodoxie religieuse et par leur affiliation avec un leader spirituel vivant le plus souvent en Israël ou aux États-Unis. Plusieurs de 
ces communautés possèdent leur propre lieu de culte, leurs écoles (pour les filles et les garçons) et leurs groupes d'études religieuses pour les hommes. Les Juifs hassidiques sont des Juifs « religieux » (le terme hassid signifie « pieux » en Hébreu), c'est-à-dire vivant le plus possible en accord avec les 613 lois du judaïsme et dans le respect des traditions religieuses et culturelles particulières du mouvement hassidique esteuropéen. Les membres de cette communauté (surtout les hommes et les garçons) peuvent être considérés comme faisant partie des minorités dites « visibles »8, leur apparence physique (barbe et papillotes, chapeau, vêtements traditionnels, etc.) étant étroitement liée à leur affiliation religieuse. De façon générale, les communautés hassidiques possèdent leurs propres institutions sociales (écoles, lieux de culte, etc.) qui se distinguent de celles de la communauté juive montréalaise organisée. Ils se caractérisent donc par une certaine autonomie du point de vue de l'organisation sociale et communautaire (Anctil, 1997) ; ils utilisent par contre plusieurs services qui ne leur sont pas exclusifs, comme les soins de santé (CLSC, hôpitaux, cliniques, etc.), des installations récréatives et sportives, des commerces de proximité (pharmacie, magasin de vêtements, garage, quincaillerie...), transport en commun, etc.

\section{Présentation des cas à l'étude}

Les cas que nous avons étudiés concernent deux synagogues hassidiques dans le secteur Mile-End/Outremont s'étant retrouvées au centre de controverses impliquant des résidants, des membres de la communauté hassidique, des représentants municipaux et les médias. Notre premier cas est celui d'une synagogue installée « illégalement » sur la rue Durocher, au rez- de-chaussée d'un immeuble résidentiel dont le zonage n'était pas approprié pour ce type d'activité. Notre deuxième cas est celui de la synagogue de la communauté Belz sur la rue JeanneMance, qui s'est adressée à la Ville de Montréal en 2001 pour obtenir un troisième permis d'agrandissement pour ses installations.

Le premier cas, qui implique la communauté hassidique «Amour pour Israël9 », a fait considérablement de bruit, surtout dans les médias locaux et à l'hôtel de ville d'Outremont, et a engendré des tensions importantes entre les différents partis (Anctil, 1997). Cette communauté hassidique avait déjà été au centre d'une autre importante controverse à la fin des années 1980 lorsqu'elle avait cherché à obtenir un changement de zonage afin de pouvoir construire une nouvelle synagogue sur la rue Saint-Viateur (une artère mixte résidentielle-commerciale). Face à l'opposition manifestée par plusieurs résidants du secteur lors de l'assemblée du conseil municipal, celui-ci avait décidé de ne pas aller de l'avant avec le changement de zonage proposé. La congrégation s'est par la suite installée au rez-de-chaussée d'un immeuble résidentiel sur la rue Durocher, dans un local pouvant accueillir des activités commerciales. Toutefois, pour des raisons techniques, le droit acquis par rapport aux activités commerciales fut révoqué et la synagogue s'est retrouvée en situation d'irrégularité par rapport au règlement de zonage de la ville d'Outremont. Une résidante de l'immeuble, après des plaintes répétées auprès de la Ville qui n'est pas intervenue pour faire déloger la synagogue, a décidé de prendre la situation en main et a intenté une poursuite en cour supérieure contre la communauté hassidique «Amour pour Israël »10. D'autres riverains et citoyens d'Outremont étaient également opposés à la présence de la synagogue sur la rue Durocher, et ce conflit de zo- 
nage est devenu une occasion de ventiler diverses frustrations vécues de part et d'autre par les citoyens. En somme, certains dénonçaient les «privilèges » que l'administration municipale accorderait aux communautés juives d'Outremont, notamment parce qu'elle n'intervenait pas systématiquement dans les dossiers de lieux de culte « illégaux 》 et qu'elle accordait des tolérances de stationnement durant les offices religieux ${ }^{11}$. La Ville n'agirait pas non plus pour faire suite aux plaintes des riverains concernant le bruit ou l'obstruction à la circulation résultant de certaines activités des communautés hassidiques.

Notre deuxième cas, celui de la synagogue de la communauté hassidique Belz de la rue Jeanne-Mance dans le quartier montréalais du Mile-End, est différent du premier en ce que les Belz sont installés au même emplacement depuis le début des années 195012. À l'origine, la congrégation hassidique avait acheté un duplex qui servait déjà de synagogue à une autre communauté juive orthodoxe. En 1971, les Belz se sont portés acquéreurs d'une deuxième maison adjacente, qu'ils ont aménagée pour agrandir la synagogue existante. En 1988, ils faisaient l'acquisition d'une troisième maison, puis d'une quatrième en 1999. À chaque étape de cette expansion, la Ville de Montréal a accordé une permission spéciale à la communauté Belz, lui permettant de transformer et d'occuper ces maisons à des fins cultuelles. L'opposition des riverains à l'expansion continue de la synagogue a commencé à se manifester en 1988 lors de l'achat par la congrégation de la troisième maison ; les plans pour cet agrandissement comprenaient aussi une importante extension vers l'arrière du bâtiment. Des voisins, rassemblés sous la bannière du Comité de rue Jeanne-Mance, ont alors fait entendre à l'administration municipale qu'ils ne désiraient pas que la synagogue puisse s'agrandir davantage. Ils ont fait valoir que la présence de cette institution au milieu d'une rue entièrement résidentielle causait plusieurs désagréments associés, entre autres, au va-et-vient d'un grand nombre de personnes plusieurs fois par jour et à la circulation accrue d'autobus et d'automobiles. La Ville a quand même décidé d'accorder le permis d'agrandissement à la congrégation Belz, mais en posant certaines conditions concernant notamment l'installation d'un système de ventilation qui permettrait de maintenir les fenêtres fermées, l'aménagement d'un parterre devant la synagogue afin de conserver une apparence résidentielle, et la résolution des problèmes de stationnement. Bien que la condition principale de cette entente, qui voulait que la congrégation ne puisse demander à la Ville d'agrandir à nouveau avant dix ans, ait été respectée, plusieurs autres conditions n'ont pas été remplies. Cette situation explique en partie pourquoi le Comité de la rue Jeanne-Mance s'est fermement opposé au nouveau projet d'agrandissement de la communauté suite à l'achat de la quatrième maison en 1999. Malgré l'opposition des riverains et le fait que le Service du développement économique et urbain et la Commission de développement urbain de la Ville n'aient pas recommandé le projet, le conseil municipal adoptait néanmoins en 2001 le règlement qui accordait une nouvelle permission spéciale à la congrégation Belz pour l'agrandissement de leur synagogue.

\section{Les contextes locaux}

Bien que leurs contextes respectifs soient fort différents ${ }^{13}$, ces deux situations sont similaires à plusieurs points de vue : elles impliquent toutes deux des communautés hassidiques installées depuis longtemps dans le quartier et des résidants locaux qui contestent la 
présence (à Outremont) et l'agrandissement (dans Mile-End) de ces lieux de culte. Les griefs exprimés par les riverains sont également similaires : la présence de ces lieux de culte dans des environnements exclusivement résidentiels entraînerait des « nuisances » telles que l'augmentation de la circulation automobile, des problèmes de stationnement, le passage fréquent d'autobus, du bruit (chants, systèmes de ventilation, conversations des fidèles avant et après les offices...), etc. Ces synagogues, qui servent à la fois pour la prière, les études religieuses, la célébration de fêtes religieuses et d'autres activités communautaires, sont fréquentées relativement intensivement lors du Sabbat hebdomadaire (bien que souvent moins en semaine). Outre les inconvénients résultant directement de la présence de ces lieux de culte, plusieurs personnes craignent que cette présence puisse avoir un effet négatif sur la valeur de leurs maisons. Aux dires de certains riverains, les immeubles dans lesquels se situent les lieux de culte auraient tendance à être mal entretenus et en mauvais état et les terrains, négligés et malpropres. Ces commentaires ont aussi été formulés à l'endroit des demeures de familles hassidiques, qui sont facilement identifiables en raison notamment de la mezouza apposée sur le cadre de la porte ${ }^{14}$. Paradoxalement, la présence hassidique dans le secteur semble avoir l'effet contraire sur le prix des maisons : la concurrence croissante sur le marché immobilier entre certaines catégories d'acheteurs (dont les gentrificateurs d'origine européenne ou canadienne) et des familles juives hassidiques aurait plutôt tendance à faire gonfler les valeurs des résidences du quartier. $\mathrm{Ce}$ phénomène n'est pas étranger à la présence des synagogues et institutions juives qui font en sorte que les familles juives hassidiques et ultraorthodoxes cherchent à demeurer dans le secteur.
Enfin, dans les deux cas que nous avons étudiés, la majorité des résidants locaux à qui nous avons parlé observent que les Juifs hassidiques entretiennent peu ou pas de contacts avec des personnes ou des organismes à l'extérieur de leur propre communauté. Ces affirmations reflètent des points de vue souvent exprimés dans les médias au sujet des Juifs hassidiques, c'est-à-dire que ceux-ci vivraient en marge de la société québécoise, qu'ils ne salueraient pas leurs voisins, qu'ils éviteraient tout contact avec les personnes à l'extérieur de leur communauté, qu'ils ne se préoccuperaient pas des autres communautés avec lesquels ils coexistent, etc. Bref, les relations entre les Juifs hassidiques et les « autres » (groupes ou individus) dans le secteur Mile-End et du basOutremont font l'objet d'un grand nombre de perceptions négatives et, bien que les confrontations ouvertes soient rares, des tensions et des préjugés semblent exister de part et d'autre. II semble aussi que ces perceptions et sentiments se cristallisent à propos de l'aménagement des lieux de culte. En effet, ces situations de tension permettent d'exprimer de façon socialement acceptable un certain malaise vis-à-vis de l'autre, c'est-à-dire en utilisant des critères « objectifs » tels que le bruit ou les problèmes de stationnement.

\section{LES ENJEUX DE LA COHABITA- TION : DIMENSION URBAINE}

\section{Le jeu du marché et du nombre}

Les tensions entourant l'aménagement des synagogues hassidiques dans nos secteurs d'étude renvoient aussi à des enjeux territoriaux : la communauté hassidique connaît une importante croissance démographique, mue par un taux de natalité nettement plus élevé que celui du reste de la population; la taille des ménages des familles has- 
sidiques et ultraorthodoxes, estimée à 5,06 personnes en moyenne ${ }^{15}$, contraste ainsi fortement avec le profil démographique du secteur. En somme, lorsqu'une communauté prend de l'expansion, elle tend à prendre plus de place, et d'autres groupes ou individus peuvent craindre de perdre la leur... Les témoignages de résidants et résidantes du secteur dans l'excellent documentaire de Gary Beitel, « Bonjour! Shalom! », ainsi que ceux recueillis lors de nos entretiens, illustrent bien cette crainte. Cet enjeu territorial s'exprime principalement à travers la compétition pour le logement ; en effet, le bas-Outremont et le quartier Mile-End sont des quartiers de plus en plus prisés par certaines catégories de gentrificateurs anglophones et francophones. En conséquence, le nombre d'appartements disponibles sur le marché local diminue, et les prix de loyers augmentent (comme c'est le cas partout sur le Plateau Mont-Royal actuellement). La compétition est parfois féroce pour accéder aux logements disponibles, à vendre ou à louer. On se coopte d'ailleurs entre soi pour mettre la main sur une propriété à vendre, tant du côté des gentrificateurs (Germain et al., 1995) que de celui de la communauté hassidique. Étant donné ce contexte, on comprend que l'aménagement de lieux de culte dans des immeubles résidentiels provoque aussi des réactions.

Dans une moindre mesure, cette compétition pour l'espace se vit aussi dans certains lieux publics ou semi-publics du secteur, notamment les trottoirs, les rues et ruelles, de même que certains mini-parcs urbains. Par exemple, quelques résidantes s'indignent du fait que les Juifs hassidiques « monopolisent » les trottoirs et ne se « tassent » pas pour laisser passer les autres (qu'il s'agisse d'hommes en route vers la synagogue ou de femmes avec poussette et plusieurs enfants), qu'ils " envahissent » la rue et l'espace public à l'oc- casion de certaines fêtes religieuses ou célébrations (mariages, visite d'un grand rabbin, etc.), sans parler des " attroupements » autour de la synagogue et des voitures stationnées un peu partout en semaine. Dans le cas des ruelles ou des mini-parcs, certaines personnes nous ont fait part de ce qu'elles perçoivent comme l'omniprésence des enfants Juifs hassidiques et de leur appropriation parfois " agressive » des aires de jeu... Même si, comme le souligne Gary Beitel, les personnes fortement négatives à l'égard des Juifs hassidiques représentent une minorité de la population outremontaise, ce sont néanmoins souvent leurs opinions qui sont repris dans les médias. De plus, les débats récents au sujet d'erouvs 16 installés par la communauté juive hassidique nourrissent cette situation de tension entre les Juifs hassidiques et certains autres résidants du secteur, notamment ceux associés au mouvement de promotion de la laïcité. Quoi qu'il en soit, la présence de plus en plus importante (et affirmée, diront certains) des Juifs hassidiques dans le secteur et la réaction correspondante de certains « autres » engendrent des tensions et transparaissent de plus en plus dans les modalités de coexistence entre ces groupes à l'échelle locale.

\section{Visibilité et marquage " ethnique » de l'espace}

La visibilité et le marquage « ethnique » de l'espace sont des éléments significatifs pour comprendre la dynamique territoriale des différents groupes dans nos secteurs d'étude. Comme nous l'avons mentionné en introduction, les hommes et les garçons de la communauté hassidique sont facilement identifiables en raison de leur tenue vestimentaire et de certains attributs physiques, dont la barbe et les papillotes. Les femmes se distinguent moins, 
mais leur tenue vestimentaire modeste, le port usuel de la perruque (sheit) ou d'un foulard recouvrant entièrement les cheveux font en sorte qu'elles soient assez faciles à identifier. Un autre facteur qui distingue les Juifs hassidiques est sonore plutôt que visuel : la langue. En effet, la grande majorité des membres, jeunes ou vieux, de cette communauté parlent Yiddish, la langue germanique traditionnelle des Juifs d'Europe de l'est et la lingua franca du hassidisme. Ces deux éléments caractéristiques, l'apparence extérieure et la langue, servent à démarquer les Juifs religieux des autres groupes culturels. II semble aussi que ces codes culturels servent aussi (et surtout) à distinguer les Juifs hassidiques des autres Juifs, moins orthodoxes ou sécularisés. En effet, les vêtements et les attributs physiques font en sorte que les Juifs religieux puissent se reconnaître entre eux, et à leur rappeler leur mode de vie spécifique. D'une certaine façon, l'habit traditionnel « protège » la spécificité culturelle et religieuse des Juifs hassidiques en les « isolant » du monde extérieur et de ses influences.

Du point de vue spécifiquement urbain, il existe un certain marquage « ethnique » associé à la communauté juive hassidique qui aurait aussi pour fonction de servir de repère aux membres de la communauté. En effet, les signes visibles de la communauté juive (outre la présence de ses membres) sont plutôt discrets : leurs lieux de culte ou les écoles s'insèrent dans la trame résidentielle existante avec un minimum d'ornementation ou d'affichage extérieurs. Les commerces sont souvent effacés derrière une vitrine typiquement encombrée, les enseignes et affiches sont souvent soit absentes ou rédigées en yiddish ou en hébreu traditionnel (du moins dans les établissements fréquentés quasi exclusivement par les membres de la communauté). Enfin, les résidences des Juifs religieux affichent sou- vent la mezouza traditionnelle ou encore des ornements sur la porte extérieure gravés d'une bénédiction en hébreu. Ces marqueurs utilisent des codes culturels qui ne semblent pas orientés vers l'extérieur de la communauté, soit parce qu'ils utilisent la langue spécifique du groupe ou que leur signification symbolique réfère à des origines ou traditions culturelles très spécifiques. Cette situation contraste avec les stratégies de visibilisation utilisées par d'autres groupes, comme les communautés islamiques montréalaises étudiées par Daher (1998), ou celles des communautés italienne ou chinoise dans leurs enclaves commerciales ${ }^{17}$. Dans le cas des communautés musulmanes plus récemment établies au Canada et au Québec, cette stratégie de visibilité chercherait à établir une certaine reconnaissance politique de ce groupe et passe par la demande pour des institutions islamiques reconnues par les gouvernements, dont des lieux de culte (dans certains cas avec minaret et autres formes architecturales à contenu symbolique). Pour d'autres communautés plus anciennes, comme les Italiens ou les Chinois (voire même les Asiatiques de façon plus générale), leur visibilité s'appuie en grande partie sur la présence physique d'une enclave commerciale dont les boutiques offrent une marchandise soit de proximité (restaurants, épiceries, etc.) ou à caractère touristique ou exotique. Alors que la Petite Italie ou le quartier chinois sont des espaces situés dans des secteurs commerciaux ou mixtes du point de vue des fonctions urbaines et qu'ils sont relativement ouverts aux membres d'autres groupes culturels, ceux des Juifs hassidiques sont, à quelques exceptions près, quasi-exclusivement fréquentés par les membres de cette communauté. Non pas que les autres en soient délibérément exclus, mais le caractère spécifique de ces endroits peut ne pas paraître « invitant » pour la communauté extérieure. Les com- 
merces ou entreprises appartenant à des membres de la communauté hassidique et offrant des services moins « ethnospécifiques » (manufactures, import/export, électronique, etc.) sont moins souvent des services de proximité et sont, de plus, situés à l'extérieur des secteurs résidentiels de cette communauté. Par contre, les membres de la communauté hassidique fréquentent régulièrement les commerces locaux et certains endroits publics du secteur, dont les pharmacies, quincailleries, les commerces de variétés ou de vêtements, les parcs, les cliniques médicales, etc. De cette façon, il existe peu d'occasions pour les personnes extérieures à la communauté juive religieuse de prendre part à la sociabilité publique qui se déroule dans les commerces fréquentés presque exclusivement par ce groupe ou leurs institutions sociales et religieuses ; cette réalité contribue sans doute à nourrir un sentiment d'exclusion ou même de rejet dont nous ont fait part certains voisins ou résidants du secteur. Assez curieusement, les exemples positifs de contacts sont peu évoqués, bien qu'ils existent. En effet, il arrive souvent que des familles hassidiques et des familles non-juives aient de bonnes, voire de très bonnes, relations de voisinage ; on se salue, on s'invite à l'occasion (surtout entre femmes), on se rend de petits services. Autant d'exemples de bon voisinage qui sont, le plus souvent, passés sous silence ou éclipsés par des exemples négatifs ou des préjugés dans le discours plus général.

\section{L'obligation de prière et la}

\section{proximité}

Il faut dire que les rapports à l'espace urbain sont également très différents de part et d'autres ; d'une part, le mode de vie des communautés hassidiques du
Mile-End/bas-Outremont est fortement dépendant de la proximité résidentielle des membres, ainsi que de la proximité des institutions sociales (écoles, lieux de culte) et services spécialisés (notamment par rapport à l'alimentation). En effet, les hommes se rendent généralement deux fois par jour à la synagogue pour effectuer les prières quotidiennes, alors que les femmes et les enfants s'y rendront surtout pour le Sabbat hebdomadaire (du vendredi soir au samedi soir). Lors de ce jour de repos, les Juifs observants ne peuvent utiliser leur voiture ni se déplacer au-delà d'une certaine distance, ce qui explique la nécessité pour leurs lieux de culte d'être situés à distance de marche. En ce qui concerne les femmes, leur vie quotidienne déborde rarement des limites du secteur18 : les écoles des enfants sont situées à proximité, elles font leurs courses à pied (souvent accompagnées de leurs enfants d'âge préscolaire), socialisent entre elles, amènent les enfants au parc ; la plupart ne conduisent d'ailleurs pas d'automobile ${ }^{19}$. Enfin, la vie communautaire intense des Juifs hassidiques, ainsi que la spécificité de leur mode de vie religieux, font que la contiguiité résidentielle est très importante. Plusieurs personnes à qui nous avons parlé dans la communauté hassidique ont fait état des défis que pose le maintien de leur mode de vie dans une société " séculière » aux valeurs « libérales »; la présence d'autres familles aux valeurs similaires (surtout d'autres familles hassidiques) permet en quelque sorte de "s'isoler » du monde extérieur et des tentations qu'il contient, de créer un environnement propice au maintien des traditions religieuses. En somme, le rapport à l'espace des Juifs hassidiques nous apparaît assez pragmatique : ils vivent dans ce secteur parce qu'ils y sont établis depuis leur arrivée à Montréal dans les années 1950, et que leurs institutions sociales essentielles s'y sont enracinées. II est difficilement concev- 
able de déménager ailleurs, car ceci impliquerait le déplacement non seulement d'un grand nombre de familles, mais aussi des institutions 20 .

D'autre part, le bas-Outremont et le quartier Mile-End présentent un profil de population assez similaire, caractérisé entre autres par une certaine mixité des origines culturelles. Depuis les années 1980, maints duplex et triplex du secteur ont été investis et rénovés par des « gentrificateurs » d'origine québécoise ou européenne. Germain et al. (1995 : 297) décrivent l'ambiance du quartier Mile-End comme « une sorte de compromis entre une urbanité à l'européenne (on se salue et on s'invite) et une relative incivilité publique nordaméricaine ». On y retrouve des éléments qui renvoient à ce que certains conçoivent comme une véritable « vie de quartier »: avec sa trame résidentielle dense et serrée doublée d'une armature commerciale très achalandée, le Mile-End « favorise le croisement des trajectoires quotidiennes car les occasions de rencontre y sont nombreuses » (Germain et al., 1995 : 271). En effet, surtout sur la rue Saint-Viateur, la présence de nombreux commerces de proximité à saveur « ethnique » ou « européenne » encourage la fréquentation quotidienne et la sociabilité publique. Les commerces, les nombreux cafés et la rue elle-même servent à soutenir cette sociabilité, peut-être pour compenser le manque d'équipements collectifs (outre le YMCA du Parc) ou d'espaces verts. Dans une moindre mesure, cette ambiance caractérise aussi le bas-Outremont, à la différence que dans le Mile-End le cosmopolitisme et la diversité culturelle sont « mis en scène » et valorisés beaucoup plus que dans le bas-Outremont. En témoigne la fête multiculturelle de la Saint-Jean-Baptiste organisée chaque année dans le Mile-End et qui se veut une célébration des nombreuses cultures qui se côtoient sur son territoire.
Si la diversité culturelle est un trait marquant de ces deux secteurs, il semble néanmoins que la diversité sur le plan socio-économique s'efface progressivement avec le degré d'avancement du processus de gentrification. Quelques personnes à qui nous avons parlé dans le cadre de notre recherche expliquent que, depuis cinq ou sept ans, l'accélération de la gentrification a un impact sur certains aspects de la cohabitation ; notamment les propriétaires résidentiels associés à ce phénomène auraient plus tendance à se préoccuper de la valeur de leur propriété (apparence extérieure, rénovations, aménagement paysager, propreté, etc.) et seraient peut-être moins « tolérants » à l'égard des modes de vie ou des comportements qui diffèrent des leurs. II faut dire aussi que la sociabilité publique dans le Mile-End et dans Outremont se reflète peu dans la dynamique associative, qui demeure somme toute assez fragmentée. C'est particulièrement vrai du côté d'Outremont, où ce processus de gentrification est plus ancien et la diversité culturelle, moins valorisée.

Ces observations quant à la sociabilité publique et au rapport à l'espace urbain nous amènent à constater que les communautés hassidiques et d'autres groupes de population, dont les gentrificateurs sont peut-être l'exemple extrême, ont des perceptions et des représentations fort différentes de leur espace de vie commun. Les trajectoires quotidiennes de ces deux populations se superposent d'ailleurs plus qu'elles ne s'entrecroisent ; il y a peu d'interactions entre les Juifs hassidiques et les autres dans l'espace public, ce qui renforce l'impression que ces deux groupes vivent en parallèle, s'ignorant l'un l'autre. Le parcours quotidien des premiers s'effectue généralement entre la résidence, le lieu de culte, le lieu de travail (sur place ou ailleurs), quelques commerces et parcs. Ces pôles de la vie quotidienne sont aussi concentrés 
dans l'espace, ce qui fait que certaines rues (Jeanne-Mance, Hutchison, SaintViateur) sont fortement investies alors que sur d'autres la présence des membres de ce groupe est rare (surtout du côté Mile-End où les résidences et institutions de la communauté hassidique se concentrent sur les rues Jeanne-Mance et Esplanade). II semble aussi que ces deux groupes parcourent le secteur selon des horaires différents : la vie quotidienne des Juifs religieux est réglée sur le rythme des prières journalières (généralement très tôt le matin et après la tombée de la nuit) et du calendrier des fêtes religieuses; le samedi, alors que le monde séculier bouillonne d'activités, celui des Juifs hassidiques baigne dans l'ambiance toute particulière du Sabbat hebdomadaire. Enfin, n'oublions pas non plus que si la vie quotidienne des Juifs hassidiques est fermement enracinée dans l'espace local montréalais, il n'en reste pas moins que les membres de cette communauté entretiennent des liens étroits avec d'autres pôles du judaïsme hassidique à l'échelle mondiale : ils se considèrent avant tout comme un peuple en diaspora, ce qui introduit une dimension symbolique toute particulière dans leur rapport au temps et à l'espace.

\section{EN GUISE DE CONCLUSION...}

Quels sont donc les éléments de réflexion qui se dégagent de l'étude de nos deux cas de cohabitation interculturelle ? En premier lieu, par rapport à l'aménagement des lieux de culte hassidiques, il semble clair que les débats qui y sont associés réfèrent à un contexte plus général de cohabitation et permettent l'expression de certaines frustrations de façon plus acceptable, c'est-à-dire en utilisant des critères urbanistiques « objectifs » tels que le bruit ou les problèmes de stationnement. À notre avis, les enjeux réels se situent au niveau du partage de l'espace urbain entre deux groupes qui évoluent dans des univers culturels et sociaux fort différents, et dont le profil socio-démographique contraste assez fortement. De plus, ces deux factions se concurrencent sur un marché résidentiel de plus en plus réduit et de moins en moins accessible. Alors que la communauté juive connaît une expansion florissante, ses besoins en espace (résidentiel, institutionnel, de loisirs, etc.) grandissent, ce qui inquiète les autres groupes de résidants qui se sentent souvent exclus des espaces occupés par cette communauté. Les perspectives d'avenir pour le secteur semblent néanmoins tendre vers une certaine homogénéisation : culturelle, si la population juive hassidique continue de croître et d'investir le secteur, et socioéconomique si le processus de gentrification finit par l'emporter et que « l'embourgeoisement » du secteur suit le modèle du Plateau Mont-Royal ou de certains secteurs d'Outremont. L'une ou l'autre de ces perspectives laisse présager que l'ouverture et la valorisation de la diversité pourraient se dissiper progressivement avec l'homogénéisation de la population et une plus grande recherche de «l'entre-soi ». Comme le rappelle D. Latouche (1998), « [...] l'accommodement trouve mal sa place dans les stratégies du confort où prime la volonté de nier ou de désamorcer la différence. II faut aimer l'inconfort pour apprécier l'accommodement. »

Plus récemment, les résultats des élections municipales de novembre 2001 ont, à notre avis, illustré de façon très claire les tensions particulières du contexte outremontais : la candidate indépendante Céline Forget, dont les démêlés avec certains groupes hassidiques ont été fort publicisés dans les médias locaux et régionaux, a remporté $34,47 \%$ des voix du district ; Forget est arrivée bonne deuxième derrière Claude Piquette (de l'équipe Tremblay), 
qui a remporté le poste de conseiller du district Jeanne-Sauvé (arrondissement d'Outremont) avec 35,38\% des votes et seulement une quarantaine de ballots de plus en sa faveur. En considérant que la plate-forme électorale de Mme Forget comportait nettement des éléments traduisant une volonté de durcissement vis-à-vis des communautés juives hassidiques, il semble difficile de croire qu'un processus de réconciliation ait été amorcé.

Qu'en est-il alors de la variable spécifiquement religieuse impliquée dans cette situation de cohabitation ? Nous croyons que la différence religieuse dans nos études de cas vient surtout exacerber des tensions culturelles liées au partage de l'espace ; en effet, une certaine dose de méconnaissance et de préjugés caractérise les relations entre les Juifs hassidiques et les autres résidants du secteur. Ces tensions nous apparaissent certes tributaires d'une certaine compétition pour l'espace entre deux groupes dans un contexte sociodémographique en transformation. Mais il reste que les différences basées sur l'appartenance et la pratique religieuse sont peut-être plus difficiles à concilier que d'autres formes d'altérité parce qu'elles réfèrent à des systèmes de valeurs qui sont souvent considérés comme immuables et rétrogrades. Plus largement, la question des lieux de culte renvoie à un débordement des pratiques et des appartenances considérées comme «privées » par la majorité des citoyens dans l'espace public à la fois physique et symbolique : l'espace public « physique », urbain, par la présence visible des lieux de culte et l'expression publique des appartenances religieuses dans cet espace ; et l'espace public « symbolique » que représente l'espace civique et politique municipal. À cet égard, on s'attend souvent à ce que les municipalités gèrent en fonction de ce principe de séparation entre privé et public les demandes de reconnaissance et d'accommodement formulées par différents groupes ethnoreligieux ou culturels. Le sujet de la gestion municipale des différences nous apparaît aussi comme un champ fertile d'exploration du vivre-ensemble qu'implique la diversité croissante de la population montréalaise.

En réalité, il nous semble que plusieurs aspects concrets des situations conflictuelles que nous avons étudiées pourraient faire l'objet de compromis si des voies de communication et de dialogue étaient mises en place. Toutefois, comme nous l'avons vu, il existe peu d'interaction entre la communauté hassidique et l'extérieur, et peu d'occasions de tisser des liens en dehors de ceux suscités par la proximité résidentielle. C'est peut-être à ce niveau que l'intervention d'une instance médiatrice (un organisme local, une agence municipale, un groupe communautaire, etc.) pourrait avoir un effet bénéfique.

En conclusion, la gestion de la diversité culturelle à l'échelle locale pose d'importants défis, à la fois aux résidants urbains eux-mêmes et aux administrations municipales; du point de vue de l'aménagement urbain, les politiques et les pratiques recèlent souvent des idéaux promus par le courant de pensée moderne sur la ville et la société urbaine. La spécialisation des espaces urbains et l'homogénéité des formes sont aussi associées à une certaine organisation de la société urbaine sur la base du statut socio-économique et d'un certain " confort culturel », pour reprendre l'expression de C. McNicoll (1993). L'altérité et la différence font pourtant partie intégrante de l'expérience urbaine et, comme nous le rappelle A. Giddens (1994), la vision du « quartier-village » n'est peut-être pas pleinement compatible avec la multiethnicité et le cosmopolitisme qui caractérisent les métropoles du XXlème siècle. 


\section{Notes}

1 Cette recherche s'inscrit dans le cadre d'une thèse de doctorat en études urbaines sous la direction de Annick Germain de I'INRSUrbanisation, Culture et Société. Elle fait également partie d'un programme de recherche financé par le CRSH (dirigé par A. Germain et F. Dansereau) sur l'Appropriation de l'espace et pratiques municipales de gestion de la diversité et d'une subvention du fonds FCAR sur le thème du Quartier. Nous tenons à remercier Annick Germain et Denise Helly, de I'INRS-UCS, ainsi que les évaluateurs (anonymes) du comité d'évaluation des Cahiers du GRES, pour leurs judicieux commentaires sur ce texte.

2 Nous entendons par lieu de culte minoritaire un lieu de regroupement dont la vocation principale est le culte religieux, établi et fréquenté par des personnes s'identifiant en fonction d'une ou de plusieurs variables culturelles (linguistique, nationale, ethnoreligieuse, etc.) distinctes de celles liées à l'identité canadienne, britannique ou française.

3 C'est-à-dire l'ensemble du territoire compris sur l'île de Montréal.

4 À l'exception de certains groupes protestants issus du mouvement de réforme radicale (voir Carle, 1995).

5 Le terme «ultraorthodoxe» a été souvent utilisé dans nos entretiens par les membres des communautés hassidiques étudiées dans le cadre de cette recherche; le terme établit une distinction avec les Juifs dits «orthodoxes», jugés plus «libéraux» par les Hassidim et généralement moins stricts dans le respect des traditions et des principes religieux. Dans le présent texte, le terme «ultraorthodoxe» réfère à la fois aux Juifs hassidiques, qui se distinguent notamment par leur affiliation à un maître spirituel lui-même identifié à la tradition des Rebbes (maîtres spirituels charismatiques) d'Europe de l'Est, et aux Juifs qui, tout en observant un degré aussi strict de respect des lois et traditions du judaïsme, ne sont pas affiliés à un maître spirituel particulier (notre utilisation du terme reprend celle de Shahar et al., 1997).

6 Outre le degré d'observance ou de pratique religieuse, d'autres caractéristiques, telles que l'affiliation à un leader spirituel et l'adhérence à certaines notions mystiques distinctes de la tradition juive classique, distinguent aussi les Juifs hassidiques des Juifs ultraorthodoxes ou plus séculiers (voir note précédente).

7 Exception faite de certaines communautés has- sidiques, essentiellement celles des Tash (établie à Boisbriand) et de certains groupes affiliés au mouvement Chabad Lubavitch, qui possèdent plusieurs centres communautaires et synagogues à l'extérieur de ce secteur.

8 Dans l'esprit de la définition proposée par Statistique Canada.

${ }^{9}$ Le nom «Amour pour Israël» est une francisation du nom véritable de la congrégation, Ahavath Israël. Le terme «Israël» ne renvoie pas, par ailleurs, à l'État (au sens politique) d'Israël actuel, considéré par plusieurs groupes hassidiques de façon très négative, mais plutôt au peuple juif et à la tradition juive au sens mythique.

10 Cette même riveraine, par la suite élue à titre de conseillère municipale pour le district, a ensuite intenté deux poursuites contre la ville d'Outremont afin d'obliger la municipalité à faire appliquer son règlement d'urbanisme. La première cause a été jugée irrecevable pour des raisons techniques, et le jugement prononcé dans la seconde cause a été en faveur de la municipalité.

11 Cette dernière mesure n'était pas exclusive aux communautés juives et a été abolie en 2001.

12 Noter toutefois que la communauté Amour pour Israël est présente (sous une forme ou une autre) à Outremont depuis les années 1960.

13 Pour une analyse des contextes municipaux respectifs de ces deux cas, voir Gagnon, Dansereau et Germain (2000).

14 La mezouza est une petite boîte rectangulaire contenant un extrait du texte sacré de la Torah affixée sur le côté droit du cadre de porte de la grande majorité des résidences juives hassidiques.

15 Voir Shahar et al. (1997) pour des données démographiques sur la communauté hassidique et ultraorthodoxe dans ces secteurs.

16 L'erouv est un dispositif qui sert à établir un périmètre symbolique qui permet aux Juifs pratiquants d'effectuer certaines choses habituellement interdites lors du Sabbat hebdomadaire. Cet assouplissement des règles très strictes par rapport au Sabbat, grâce à l'erouv, ne s'applique qu'à certains éléments définis de façon très précise par les autorités religieuses, tels que la possibilité de transporter de la nourriture d'une maison à l'autre ou de pousser une poussette d'enfant ou une chaise roulante afin de se rendre à la synagogue. L'erouv comme tel est habituellement un fil transparent suspendu plusieurs mètres au-dessus du 
sol.

17 Voir à ce sujet Qadeer (1997).

18 Les membres de la communauté hassidique montréalaise, y compris les femmes, entretiennent par contre des liens étroits avec d'autres groupes hassidiques ailleurs, notamment aux États-Unis (État de New York) ou en Israël. Les contacts sont fréquents et les membres (y compris les femmes et les enfants) voyagent souvent entre les différents pôles nord-américains du judaïsme orthodoxe.

19 Cette attitude traditionnelle par rapport aux femmes et la conduite automobile semble toutefois être en voie de changer et, à Montréal, quelques femmes membres de la communauté hassidique conduisent et/ou possèdent une voiture.

20 II existe quelques cas à Montréal de communautés hassidiques s'étant relocalisées en tant que groupe ; la communauté Tash, qui s'est réimplantée à Boisbriand, représente peut-être l'exemple extrême de ce phénomène (voir à ce sujet Shaffir, 1993).

\section{Bibliographie}

ANCTIL, Pierre, 1997 [1989]. « Judaïsme et orthodoxie à Montréal » dans Tur Malka. Flâneries sur les cimes de l'histoire juive montréalaise. Sillery : Septentrion, ch. 7.

CARLE, Martine, 1995. Profil des principaux groupes religieux du Québec. Québec : Ministère des Affaires internationales, de l'immigration et des communautés culturelles.

CICERI, Coryse, 1998. Le foulard islamique à l'école publique : analyse comparée du débat dans la presse française et québécoise francophone (1994-1995). Mémoire de maîtrise, Faculté des sciences de l'éducation, Université de Montréal.
DAHER, Ali, 1998. La construction de l'islamité et l'intégration des musulmans au Québec dans le discours de leurs leaders. Thèse de doctorat, département de sociologie, Université du Québec à Montréal.

GAGNON, J.E., DANSEREAU, F. \& GERMAIN, A., 2000. "Ethnic » Dilemmas ? : Religion, Diversity, and Multicultural Planning in Montréal, Canada. Communication présentée dans le cadre de la Cinquième conférence internationale Metropolis, Vancouver (C.-B.), pp. 13-18, novembre.

GERMAIN, A. (coord.), ARCHAMBAULT, J., BLANC, B., CHARBONNEAU, J., DANSEREAU, F. \& ROSE, D., 1995. Cohabitation interethnique et vie de quartier. Québec : Gouvernement du Québec, Ministère des Affaires internationales, de I'Immigration et des Communautés culturelles, collection Études et recherches, $n^{\circ} 12$.

GIDDENS, Anthony, 1994. Les conséquences de la modernité. Paris : L'Harmattan.

GRAFMEYER, Yves, 1999. « La coexistence en milieu urbain : échanges, conflits, transaction », Recherches sociologiques, $\mathrm{n}^{\circ} 1$, pp.157-176.

LATOUCHE, D., 1998. « Du bon usage de l'accommodement urbain », Géographie et cultures, n 26, pp. 2544.

MCANDREW, M., 1996. « Diversité culturelle et religieuse : divergences des rhétoriques, convergences des pratiques? ", dans Gagnon, $F$. McAndrew, M. et Pagé, M. (eds), Pluralisme, citoyenneté et éducation. Paris/Montréal : L'Harmattan, pp. 286317. 
MCNICOLL, Claire, 1993. Montréal, une société multiculturelle. Paris : Bélin.

MILOT, M. \& PROULX, J.-P., 1999. Les attentes sociales à l'égard de la religion à l'école publique : rapport de recherche. Québec : Ministère de l'Éducation.

QADEER, M., 1997. « Pluralistic Planning for Multicultural Cities. The Canadian Practice », Journal of the American Planning Association, vol. $63, n^{\circ} 4$, pp. 481-494.

SHAFFIR, Wiliam, 1993. "Separation From the Mainstream in Canada: The Hassidic Community of Tash », dans R. J. BRYM, W. SHAFFIR et M. WEINFELD (eds.), The Jews in Canada, Toronto: Oxford University Press, 126-141.

SHAHAR, C., WEINFELD, M. \& SCHNOOR, R.F., 1997. Survey of the Hassidic \& Ultra-Orthodox Communities in Outremont \& Surrounding Areas. Outremont: COHO. 